\title{
A rapid seamless method for gene knockout in Pseudomonas aeruginosa
}

\author{
Weiliang Huang and Angela Wilks ${ }^{*}$ (D)
}

\begin{abstract}
Background: Pseudomonas aeruginosa is a model organism for the study of quorum sensing, biofilm formation, and also leading cause of nosocomial infections in immune compromised patients. As such P. aeruginosa is one of the most well studied organisms in terms of its genetics. However, the construction of gene deletions and replacements in Pseudomonas aeruginosa is relatively time-consuming, requiring multiple steps including suicide vector construction, conjugation, inactivation with insertion of antibiotic resistance cassettes and allelic exchange. Even employing Gateway recombineering techniques with direct transformation requires a minimum two weeks.

Methods: We have developed a rapid streamlined method to create clean deletion mutants in P. aeruginosa through direct transformation, eliminating the need for the creation of Gateway-compatible suicide vectors. In this method, upstream and downstream sequences of the gene/locus to be deleted are amplified by polymerase chain reaction (PCR) and seamlessly fused with the linearized pEX18Tc sacB suicide plasmid by Gibson assembly. The resulting deletion plasmid is transformed into $P$. aeruginosa by an electroporation method optimized in this study. The plasmid is then integrated into the chromosome by homologous recombination, and deletion mutants are identified via $s a c B$ mediated sucrose counter-selection.

Results: The current method was employed to generate clean gene deletions of the heme assimilation system anti- $\sigma$ factor, has $S$ and the virulence regulator involving ECF system anti- $\sigma$ and $\sigma$ factors vreA and vrel, respectively. The process from plasmid construction to confirmation by DNA sequencing of the gene deletion was completed in one week. Furthermore, the utility of the method is highlighted in the construction of the vreA and vrel deletions, where the start codon of vreA and the stop codon of vrel overlap. Utilizing Gibson assembly deletion mutants were constructed with single base pair precision to generate the respective vreA and vrel deletions, while maintaining the start and stop codon of the respective genes. Overall, this method allows for rapid construction of gene deletions in $P$. aeruginosa with base pair precision.

Conclusion: This method from the construction of the suicide vector to sequence confirmation of the unmarked gene deletion can be performed in one week, without the requirement for expensive proprietary reagents or instruments. The precision of Gibson assembly and the fact the accuracy in generating the desirable construct is 95\%, makes this a viable and attractive alternative to previous methods.
\end{abstract}

Keywords: Pseudomonas Aeruginosa, Genetic knockout, Gibson assembly

* Correspondence: awilks@rx.umaryland.edu

Department of Pharmaceutical Sciences, School of Pharmacy, University of

Maryland, Baltimore, MD, USA

(c) The Author(s). 2017 Open Access This article is distributed under the terms of the Creative Commons Attribution 4.0 International License (http://creativecommons.org/licenses/by/4.0/), which permits unrestricted use, distribution, and reproduction in any medium, provided you give appropriate credit to the original author(s) and the source, provide a link to the Creative Commons license, and indicate if changes were made. The Creative Commons Public Domain Dedication waiver (http://creativecommons.org/publicdomain/zero/1.0/) applies to the data made available in this article, unless otherwise stated. 


\section{Background}

Pseudomonas aeruginosa is a common cause of hospitalacquired infections including respirator-associated pneumonias, bacteremias, and urinary tract and surgical site infections. $P$. aeruginosa accounts for 51,000 opportunistic infections/year with approximately $13 \%$ being multidrug resistant $[1,2]$. The $P$. aeruginosa genome is one of the largest bacterial genomes at approximately 6 million base pairs with about $65 \%$ guanine + cytosine content [3]. The adaptability of $P$. aeruginosa is reflected in the fact that the genome encodes 5500 open reading frames with a significant proportion of the genome dedicated to regulatory genes and genes associated with the breakdown, transport and efflux of organic compounds. The large and complex genome of $P$. aeruginosa provides unique challenges in terms of developing genetic tools by which to study the biological function of specific genes.

The generation of specific deletion mutants in $P$. aeruginosa has relied on allelic exchange by homologous recombination that requires $3-4$ weeks $[4,5]$. Hmelo et al., recently published an in-depth two-step allelic exchange protocol that can be applied to gene knockouts and knock-ins, as well as single-nucleotide insertions and deletions or substitutions [6]. The general method of two step allelic exchange requires cloning of the upstream and downstream sequences of a locus of interest into a suicide vector such as the pEX18 plasmids pioneered by the Schweizer laboratory [7]. Secondly, the deletion or mutant vector is transformed into a conjugative E. coli strain such as SM10. The mutant allele is then transferred into $P$. aeruginosa by biparental mating. The vector containing the deletion allele is integrated into the $P$. aeruginosa chromosome by in vivo homologous recombination. The resulting merodiploids are selected against the antibiotic resistance marker encoded within the integrated plasmid backbone. The isolated merodiploids undergo a second homologous recombination, which is counter-selected through the suicide marker $s a c B$ to isolate colonies that have lost the plasmid backbone. The completion of two-step allelic exchange takes upward of two weeks to complete. The use of Gateway technology to clone the mutant allele into a donor allelic exchange vector, which is then transformed into $P$. aeruginosa by electroporation, still requires at least two weeks [8].

Herein we describe an optimized and rapid method to create unmarked deletion mutants of $P$. aeruginosa in as few as seven days with total hands-on time of less than half a day. Briefly, allelic exchange constructs for gene deletion are generated by Gibson assembly [9] and introduced into $P$. aeruginosa using an optimized electroporation protocol. We have successfully created clean deletion mutants in the virulence regulator involving ECF system (vre, also known as PUMA3) and the heme assimilation system (has) of $P$. aeruginosa using this method. Herein we describe the generation of a clean deletion mutant of the anti- $\sigma$ factor receptor has $S$, the signaling receptor vreA and $\sigma$ factor vreI, respectively. We expect this method can be readily adapted to create insertion and substitution mutants in other genes and for use in related organisms.

\section{Methods \\ Media}

E. coli DH5 $\alpha$ (New England BioLabs) was maintained on lysogeny broth (LB) agar medium (10 g/L tryptone, $5 \mathrm{~g} / \mathrm{L}$ yeast extract, $5 \mathrm{~g} / \mathrm{L} \mathrm{NaCl}, 15 \mathrm{~g} / \mathrm{L}$ agar). P. aeruginosa PAO1 (obtained from Professor Michael Vasil, University of Colorado) [10] was maintained on brain-heart infusion (BHI) agar medium ( $5 \mathrm{~g} / \mathrm{L}$ beef heart infusion, $12.5 \mathrm{~g} / \mathrm{L}$ calf brains infusion, $2.5 \mathrm{~g} / \mathrm{L}$ disodium hydrogen phosphate, $2 \mathrm{~g} / \mathrm{L}$ glucose, $10 \mathrm{~g} / \mathrm{L}$ peptone, $5 \mathrm{~g} / \mathrm{L}$ sodium chloride, $15 \mathrm{~g} / \mathrm{L}$ agar). For plasmid preparation, $E$. coli transformants were cultured in terrific broth (TB) (12 g/L peptone, $24 \mathrm{~g} / \mathrm{L}$ yeast Extract, 9.4 $\mathrm{g} / \mathrm{L}$ dipotassium hydrogen phosphate, $2.2 \mathrm{~g} / \mathrm{L}$ potassium dihydrogen phosphate, $4 \mathrm{ml} / \mathrm{L}$ glycerol). Tryptone yeast extract and sucrose (TYS10) (10 g/L tryptone, $5 \mathrm{~g} / \mathrm{L}$ yeast extract, $10 \%(W / V)$ filtered sucrose, $15 \mathrm{~g} / \mathrm{L}$ agar) plates were used in the selection of the second homologous recombination event. Tetracycline at $10 \mu \mathrm{g} / \mathrm{ml}$ and $100 \mu \mathrm{g} / \mathrm{ml}$ were used to select $E$. coli transformants and $P$. aeruginosa merodiploids, respectively.

\section{Amplification of the upstream and downstream sequences of the locus of interest}

Sequence information was obtained from the Pseudomonas Genome Database (http://www.pseudomonas.$\mathrm{com} /$ ). Partial overlapping flanking primers were designed to amplify the upstream and the downstream 500 bp DNA sequences of hasS, vreI and vreA using NEBuilder (http://nebuilder.neb.com/) (Additional file 1). We describe here the construction of the hasS deletion as an example, and provide sequencing confirmation for the vreA and vreI deletion constructs (Additional file 2). The flanking primers for the has $S$ deletion construct are as follows: upstream forward, 5' cgggtaccgagctcgGCAAGACCCGCGATCCGC 3' and upstream reverse, 5' cgcg ccgcgtgcggctCTCGTTGGTC 3'; downstream forward, 5' agccgcacgcggcgcgATCGCTTCCG 3' and downstream reverse, 5' ctatgaccatgattacgGCAAGTATTCGCCGTGC ACCG 3' (overlapping bases in lower case, which facilitate fusion as shown in Fig. 1). The PCR reactions contained $200 \mu \mathrm{M}$ dNTPs, $0.5 \mu \mathrm{M}$ of forward and reverse primers, $10 \mathrm{ng} / \mu \mathrm{l} P$. aeruginosa genomic DNA, $0.02 \mathrm{U} /$ $\mu \mathrm{l}$ Q5 high fidelity DNA polymerase (New England Biolabs) in $1 \times$ reaction buffer containing $2 \mathrm{mM} \mathrm{MgCl}_{2}$ supplied by the manufacturer. The thermo cycles were programmed as follows: initial denaturation at $98{ }^{\circ} \mathrm{C}$ for 


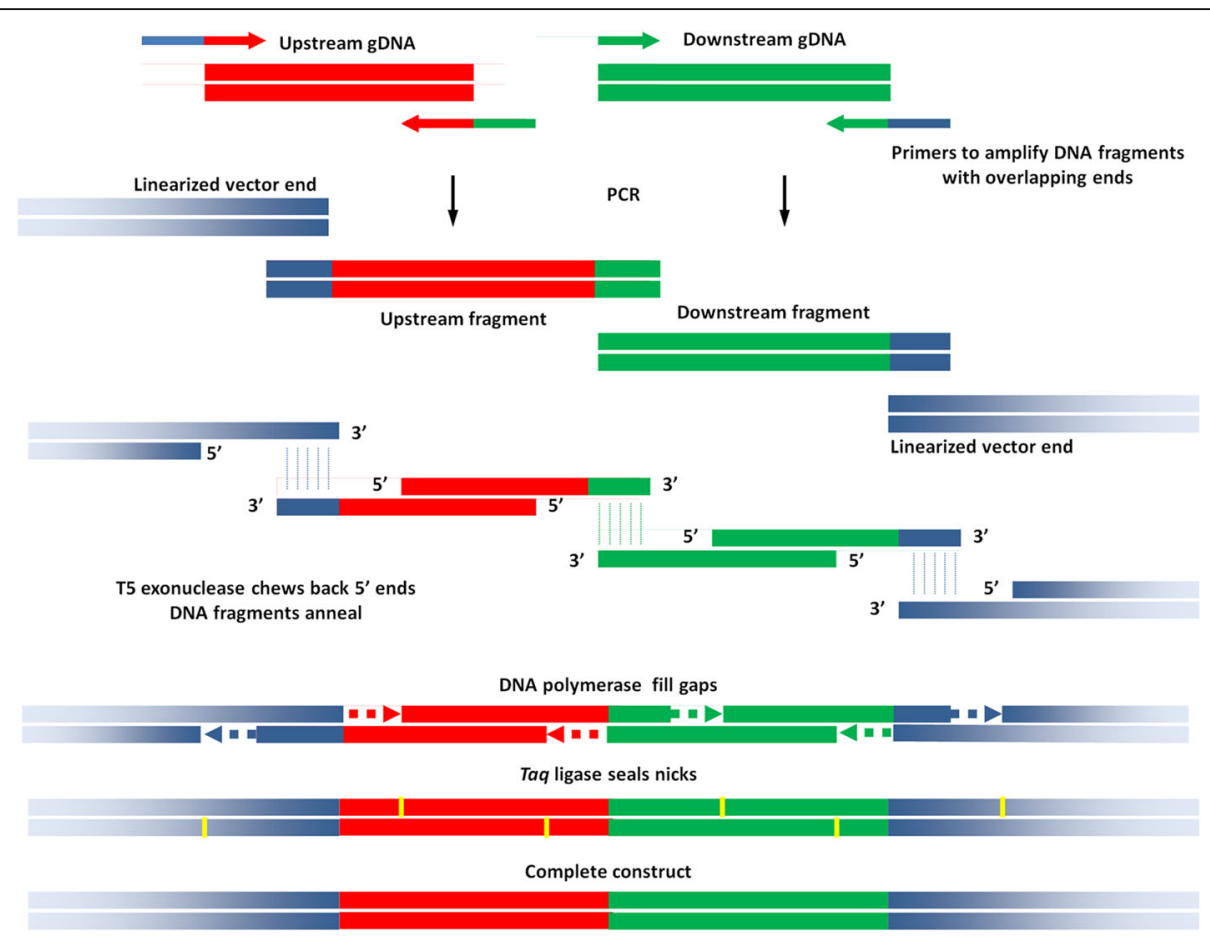

Fig. 1 Construction of deletion construct via Gibson assembly. DNA fragment ends of the same color represent identical sequences, which facilitate DNA fusion. gDNA, genomic DNA

3 min, followed by 30 cycles of $98^{\circ} \mathrm{C}$ for $10 \mathrm{~s}$, annealing temperature for $30 \mathrm{~s}$, and $72{ }^{\circ} \mathrm{C}$ for $15 \mathrm{~s}$, and a final extension at $72{ }^{\circ} \mathrm{C}$ for $2 \mathrm{~min}$. The PCR products were analyzed by DNA agarose electrophoresis and purified by QIAquick spin-column (QIAGEN) before use.

\section{Gibson assembly of the deletion construct}

The pEX18Tc suicide plasmid was linearized within the multiple cloning site by restriction digest with EcoRI. The Gibson assembly reaction was performed as described by Gibson et al. [2]. The procedure is illustrated in Fig. 1. Briefly, the reaction mix contained $10 \mathrm{nM}$ of each amplified upstream and downstream genomic DNA fragment, $5 \mathrm{nM}$ of linearized pEX18Tc plasmid, $0.004 \mathrm{U} / \mu \mathrm{l}$ of $\mathrm{T} 5$ exonuclease, $4 \mathrm{U} / \mu \mathrm{l}$ of Taq ligase, $0.0125 \mathrm{U} / \mu \mathrm{l}$ of Q5 DNA polymerase, $5 \%(W / V)$ of PEG8000, $1 \mathrm{mM}$ of NAD, $0.25 \mathrm{mM}$ of dNTPs in 1× Q5 DNA polymerase reaction buffer containing $2 \mathrm{mM} \mathrm{MgCl} 2$. Individual reagents or master mix can be acquired from New England Biolabs. The reaction mixture was incubated at $50{ }^{\circ} \mathrm{C}$ for $1 \mathrm{~h}$ and the resulting ligated plasmid was transformed into high efficiency chemically competent $E$. coli $\mathrm{DH} 5 \alpha$ (New England Biolabs). Transformants were checked by colony PCR using the universal pEX18 vector primers, which flank the multiple cloning site: forward, 5' GGCTCGTATGTTG TGTGGAATTGTG $3^{\prime}$ and reverse, 5' GGATGTGCTGC AAGGCGATTAAG 3' (annealing temperature: $55{ }^{\circ} \mathrm{C}$ ). Positive clones were further verified by DNA sequencing
(Eurofins Scientific). A colony containing the sequenced deletion suicide vector was inoculated in TB medium with $10 \mu \mathrm{g} / \mathrm{ml}$ tetracycline and shaken at $250 \mathrm{rpm}, 37^{\circ} \mathrm{C}$ for about $23 \mathrm{~h}$. Plasmid DNA was extracted by QIAprep spin miniprep kit (QIAGEN).

\section{Electroporation of $P$. aeruginosa and selection of the first} homologous recombination

A variety of electroporation conditions [11-14] were tested and a method modified on the procedure described by Choi et al. [11] was used. One fresh colony of $P$. aeruginosa PAO1 was inoculated in $5 \mathrm{ml} \mathrm{LB}$ medium and grown overnight at $42{ }^{\circ} \mathrm{C}$ without shaking until reaching stationary phase. Cells from overnight cultures $(4 \mathrm{ml})$ were harvested by centrifugation at $13000 \times \mathrm{g}$ for $1 \mathrm{~min}$ at room temperature $\left(\sim 23^{\circ} \mathrm{C}\right)$. The cell pellet was resuspended in $1 \mathrm{ml}$ of $1 \mathrm{mM}$ room temperature $\mathrm{MgSO}_{4}$ followed by centrifugation and the supernatant was discarded. The cells were washed again and the cell pellet was resuspended in $50 \mu \mathrm{l}$ of $1 \mathrm{mM}$ room temperature $\mathrm{MgSO}_{4} \cdot 5-10 \mu \mathrm{g}$ of plasmid DNA was mixed with $50 \mu \mathrm{l}$ of cell mixture, and the resulting mixture was transferred to a $2 \mathrm{~mm}$ gap electroporation cuvette (Sigma). Various pulsing parameters were tested for maximum transformation efficiency. Optimum electroporation conditions for PAO1 were determined to be $2.2 \mathrm{KV}$ with a decaying exponential waveform ( $25 \mu \mathrm{F}$ capacitor, $600 \Omega$ ) in a $2 \mathrm{~mm}$ gap cuvette at room temperature (see Results section Fig. 3). After 
pulsing on a Bio-Rad MicroPulser, $1 \mathrm{ml}$ of BHI medium was added to the cuvette immediately and the mix incubated at room temperature for $5 \mathrm{~min}$. The cells were transferred into a falcon tube and shaken at $37^{\circ} \mathrm{C}$ for $3 \mathrm{~h}$. Transformed cells were pelleted and $700 \mu \mathrm{l}$ of the supernatant was discarded. The cells were resuspended in the remaining medium and plated on two to three $\mathrm{BHI}$ agar plates containing $100 \mu \mathrm{g} / \mathrm{ml}$ of tetracycline (BHI/Tc100). The plates were incubated at $37{ }^{\circ} \mathrm{C}$ for up to $64 \mathrm{~h}$ with recombinants generally appearing within the first $48 \mathrm{~h}$.

\section{sacB counter-selection of the second homologous recombination}

Colonies growing on the BHI/Tc100 plates were checked by colony PCR using the pEX18 universal forward primer and a primer specific to the genomic DNA following the cloned $500 \mathrm{bp}$ downstream sequence $\left(5^{\prime} \mathrm{AC}\right.$ ATCTGCACCAGGTCGTC 3'), as illustrated in Fig. 4. The pEX18 universal reverse primer and a primer specific to the genomic DNA before the cloned $500 \mathrm{bp}$ upstream sequence could also be used to screen recombinants. Positive colonies were purified by streaking on new BHI/Tc100 plates. Purified merodiploids ( $2 \mathrm{~mm}$ ) were streaked on TYS10 plates using a single loop/tip and incubated at room temperature $\left(\sim 23{ }^{\circ} \mathrm{C}\right)$. Colonies that lost the integrated plasmid usually appear within $48-60 \mathrm{~h}$. Colonies were examined by colony PCR using a set of primers flanking the genomic sequence of the deleted locus hasS (forward, 5' GATTACCGAGTCT TGCCGGTTC 3', and reverse, 5' ACATCTGCACCAGGTCGTC $3^{\prime}$ ) as illustrated in Fig. 4. Colonies with the confirmed deletion were purified by streaking on a TYS10 plate and further verified by DNA sequencing (Eurofins Scientific).

\section{Results}

Rapid and accurate construction of allelic exchange vectors using Gibson assembly

The construction of the allelic exchange vector for gene has $S$ was performed as shown in Fig. 1. First, the 500 bp upstream and downstream DNA sequences flanking the locus of interest were amplified (Fig. 2a). Gibson assembly was used to fuse and clone these fragments into the pEX18Tc allelic exchange vector as described in the Materials and Methods. Other high fidelity DNA polymerase such as Phusion DNA Polymerase can also be used in the PCR and Gibson assembly. The resulting deletion construct was transformed into E. coli DH $5 \alpha$. Colonies containing the deletion construct were confirmed by colony PCR using the universal pEX18 primer pair flanking the multiple cloning sites (Fig. 2b). Positive colonies containing both the upstream and downstream DNA fragments showed a $1 \mathrm{kbp}$ insert and were further selected for DNA preparation and sequencing. The accuracy of the Gibson assembly was extremely high with more than $95 \%$ of the colonies tested during method development $(n=28)$ containing the mutant deletion allele with the correct sequence.

The pEX18 series of suicide vectors have been widely used for site-directed gene manipulation in $P$. aeruginosa and related bacteria [7]. The construction of a gene knockout vector usually involves several steps of subcloning. To accelerate the procedure, Wolfgang et al. [15] and Choi et al. [11] used overlap PCR and the Gateway cloning technology (Invitrogen) to generate gene deletion constructs. However, the Gateway technology procedure still requires several extra cloning steps: first, the upstream and downstream fragments are amplified by flanking PCR; secondly, the two fragments are fused
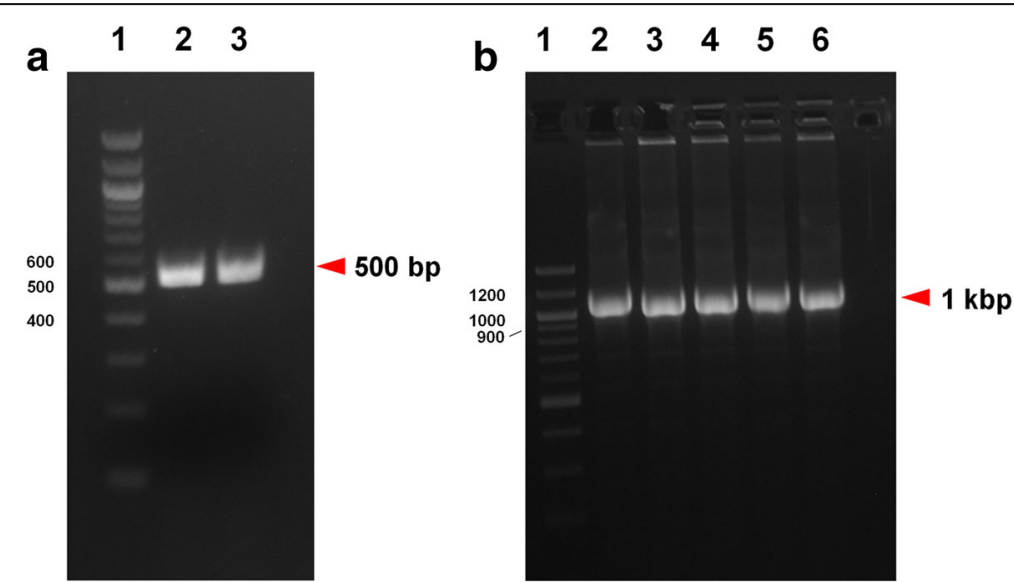

Fig. 2 a Amplification of the 500 bp upstream and downstream DNA fragments of hasS. Lane 1, 400 ng DNA ladder; lane 2, $3 \mu$ l of the 500 bp PCR product of the upstream genomic DNA sequence of hasS; lane 3, $3 \mu \mathrm{l}$ of the $500 \mathrm{bp} \mathrm{PCR} \mathrm{product} \mathrm{of} \mathrm{the} \mathrm{downstream} \mathrm{genomic} \mathrm{DNA} \mathrm{sequence} \mathrm{of} \mathrm{hasS.}$ b Colony PCR of fused deletion alleles. The $500 \mathrm{bp}$ upstream DNA fragment and the $500 \mathrm{bp}$ downstream DNA fragment were fused and cloned into the pEX18Tc vector via Gibson assembly. Lane 1, 400 ng, 100 bp DNA ladder; lane 2-6, $10 \mu \mathrm{l} \mathrm{of} \mathrm{PCR} \mathrm{products} \mathrm{of} \mathrm{five} \mathrm{colonies} \mathrm{randomly} \mathrm{selected,}$ which all showed the $1 \mathrm{kbp}$ fused deletion alleles 
together by overlap PCR; and finally the resulting deletion allele is purified by electrophoresis and cloned into a Gateway allelic exchange vector. Furthermore, the pEX18 suicide vectors must be modified to be compatible with the Gateway cloning system by adding the att flanking sites. Gateway proprietary reagents such as Clonase are used to recombine the deletion allele from the Gateway vector into the modified Gateway compatible pEX18 vector. Utilizing the method described herein the complete construct can be generated in two steps: first, the upstream and downstream DNA fragments are amplified by PCR; then the DNA fragments are ligated directly into a linearized pEX18 plasmid by single step Gibson assembly to generate the allelic exchange vector. Neither proprietary reagents nor modifications to the suicide vector are required.

\section{Quick deletion construct transfer by electroporation and merodiploid selection}

The transfer of the allelic exchange plasmid into P. aeruginosa is traditionally performed via conjugation. Specifically, the deletion construct is transformed into a conjugative $E$. coli strain, which is subsequently mated with recipient $P$. aeruginosa cells. After mating, transformed $P$. aeruginosa cells are isolated from the conjugation mixture by plating on media containing both triclosan and the antibiotic compatible with the plasmid resistance marker. Repetitive passage of the deletion

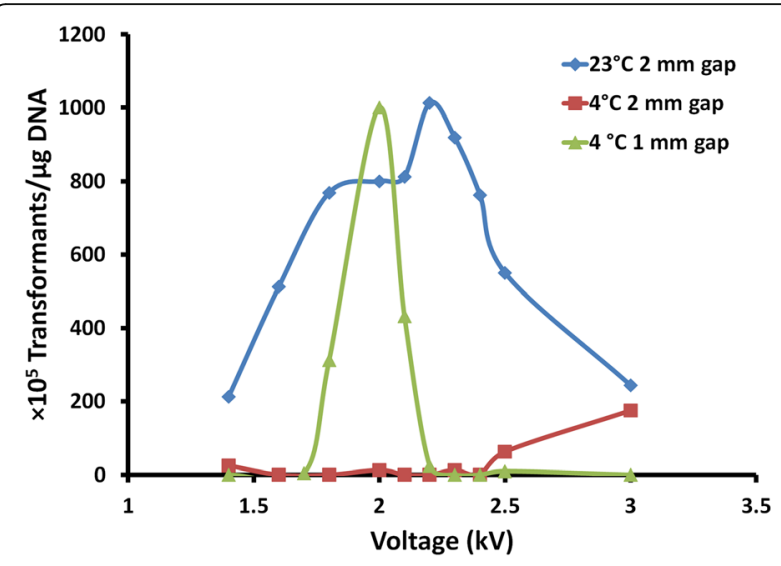

Fig. 3 The transformation efficiency of $P$. aeruginosa PAO1 with PBSP11T $c^{R}$ under various electroporation conditions. The maximum efficiency was achieved at $2.0 \mathrm{kV}$ using a $1 \mathrm{~mm}$ gap cuvette at $4^{\circ} \mathrm{C}$ or at $2.2 \mathrm{kV}$ using $2 \mathrm{~mm}$ cuvette at $23^{\circ} \mathrm{C}$. The field strength applied at $4{ }^{\circ} \mathrm{C}$ in a $1 \mathrm{~mm}$ gap cuvette is $20 \mathrm{kV} / \mathrm{cm}$. Since the membrane permeation voltage is doubled at $4^{\circ} \mathrm{C}$ as compared to room temperature [17], the field strength at $23^{\circ} \mathrm{C}$ in a $2 \mathrm{~mm}$ cuvette $(11 \mathrm{kV} / \mathrm{cm})$ is equivalent to $22 \mathrm{kV} / \mathrm{cm}$ at $4{ }^{\circ} \mathrm{C}$. Therefore, the optimum field strength is determined to be $20-22 \mathrm{kV} / \mathrm{cm}$ ( $4^{\circ} \mathrm{C}$ equivalent). Since the $2 \mathrm{~mm}$ gap cuvette is less prone to arcing, electroporation at $2.2 \mathrm{kV}$ using a $2 \mathrm{~mm}$ gap cuvette at room temperature was used to transform the suicide pEX18Tc allelic exchange construct into $P$. aeruginosa PAO1. Pulse durations of 4.0 to $4.5 \mathrm{~ms}$ were used in most experiments construct under harsh double antibiotic selection places additional stress on the cells and may promote undesirable spontaneous mutations. Alternatively, we pursued a more direct approach via electroporation that avoids the multiple steps that may cause spontaneous mutations while shortening the protocol. Although transformation of $P$. aeruginosa by electroporation methods has previously been considered relatively inefficient, we have optimized the electroporation protocol described herein to transformation efficiencies similar to those obtained by conjugation.

To inhibit the DNA restriction-modification system of $P$. aeruginosa, the cells were grown at $42-43{ }^{\circ} \mathrm{C}$ overnight without shaking as described by Rolfe et al. [10]. To optimize the transformation efficiency, a panel of electroporation solutions was tested. Significant cell lysis was observed when water, 10-15\% glycerol, HEPES buffer, $300-500 \mathrm{mM}$ sucrose, $300-500 \mathrm{mM}$ sorbitol or various combinations thereof were used. $\mathrm{MgSO}_{4}$ at $1 \mathrm{mM}$ was found to effectively maintain cell structure integrity and not interfere with electroporation. Therefore, cells were harvested and washed in $1 \mathrm{mM} \mathrm{MgSO}_{4}$ at either $4{ }^{\circ} \mathrm{C}$ or $23{ }^{\circ} \mathrm{C}$.

The optimum electric parameters were investigated by testing the transformation efficiency of a $P$. aeruginosa replicative plasmid pBSP11Tc ${ }^{\mathrm{R}}$ at both $4{ }^{\circ} \mathrm{C}$ and $23{ }^{\circ} \mathrm{C}$ using $1 \mathrm{~mm}$ gap and $2 \mathrm{~mm}$ gap cuvettes at different voltages. The efficiencies of electroporation under these conditions are shown in Fig. 3.

Using these conditions the deletion construct was transferred into $P$. aeruginosa cells and integrated into the PAO1 chromosome by homologous recombination as shown in Fig. 4. Merodiploids in which the deletion construct is integrated into the chromosome usually appeared on the tetracycline selection plates within $40 \mathrm{~h}$. In some cases, positive colonies appeared up to $60 \mathrm{~h}$ after electroporation. Beyond $72 \mathrm{~h}$, spontaneous tetracycline resistant colonies began to appear. Suicide pEX18 vector constructs with alternate antibiotic resistance markers such as carbenicillin and gentamicin were also tested. However, colonies with spontaneous resistance against these antibiotics appeared as early as $20 \mathrm{~h}$, which made selection of merodiploids more difficult. Therefore, tetracycline resistance appears to provide the most optimal selection marker for merodiploids by this method.

Tetracycline resistant colonies were analyzed by colony PCR to confirm the generation of merodiploids (Fig. 5). Using the universal pEX18 vector forward primer (primer 1 ) and a primer specific to a genomic sequence after the 500 bp downstream sequence (primer 4), colonies in which the plasmid integrated via the upstream homologous sequence yielded $\mathrm{a} \sim 2 \mathrm{kbp}$ PCR product (Fig. 5, lane 3). Meanwhile, colonies in which the plasmid integrated via the downstream homologous sequence yielded $\mathrm{a} \sim 1$ kbp band (Fig. 5, lane 2). The approximate crossovers for 


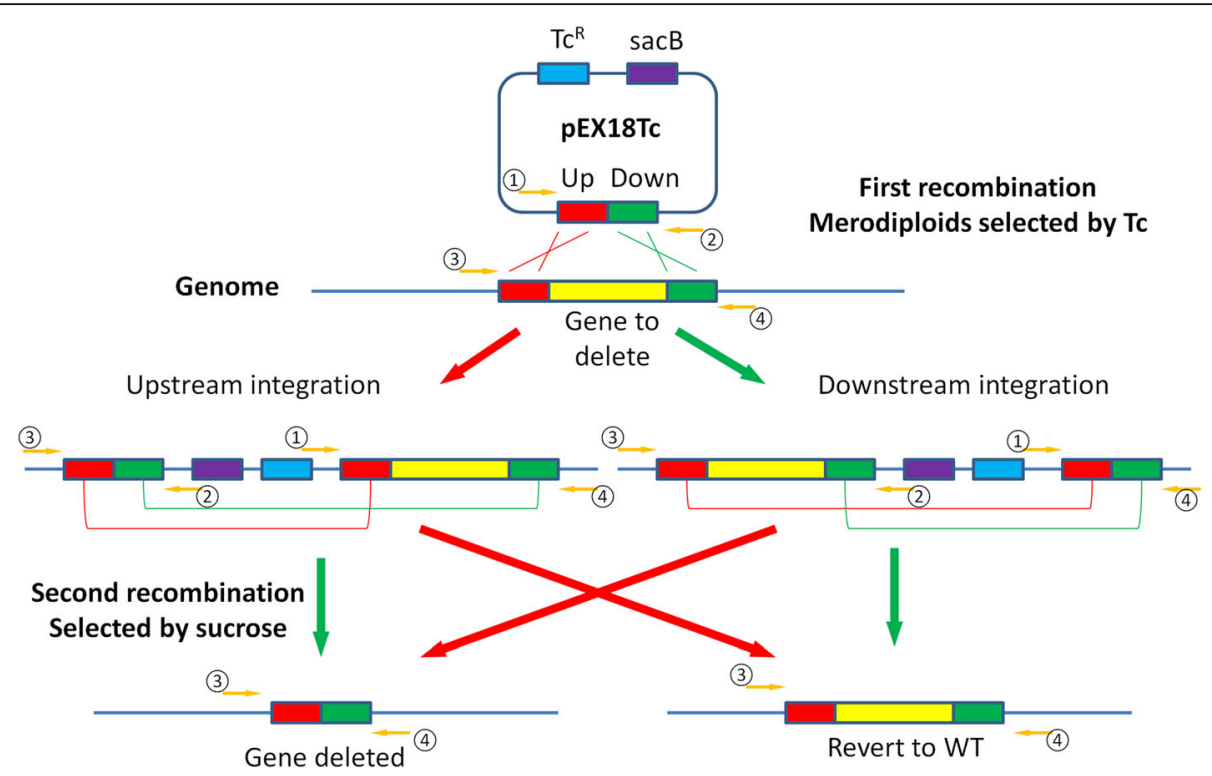

Fig. 4 Gene knockout of $P$. aeruginosa via homology recombination. Primer 1 and 2, universal pEX18 forward and reverse primers flanking the multiple cloning sites, respectively. Primers 3 and 4 , specific to genomic DNA sequences flanking the sequences cloned into the pEX18Tc vector. Primers 1 and 2 were used to screen E. coli DH5a transformants containing the deletion allele plasmid. Primers 1 and 4 or primers 2 and 3 were used to screen merodiploids in which the deletion construct was integrated into the chromosome. Primers 3 and 4 were used to screen deletion mutants following secondary recombination

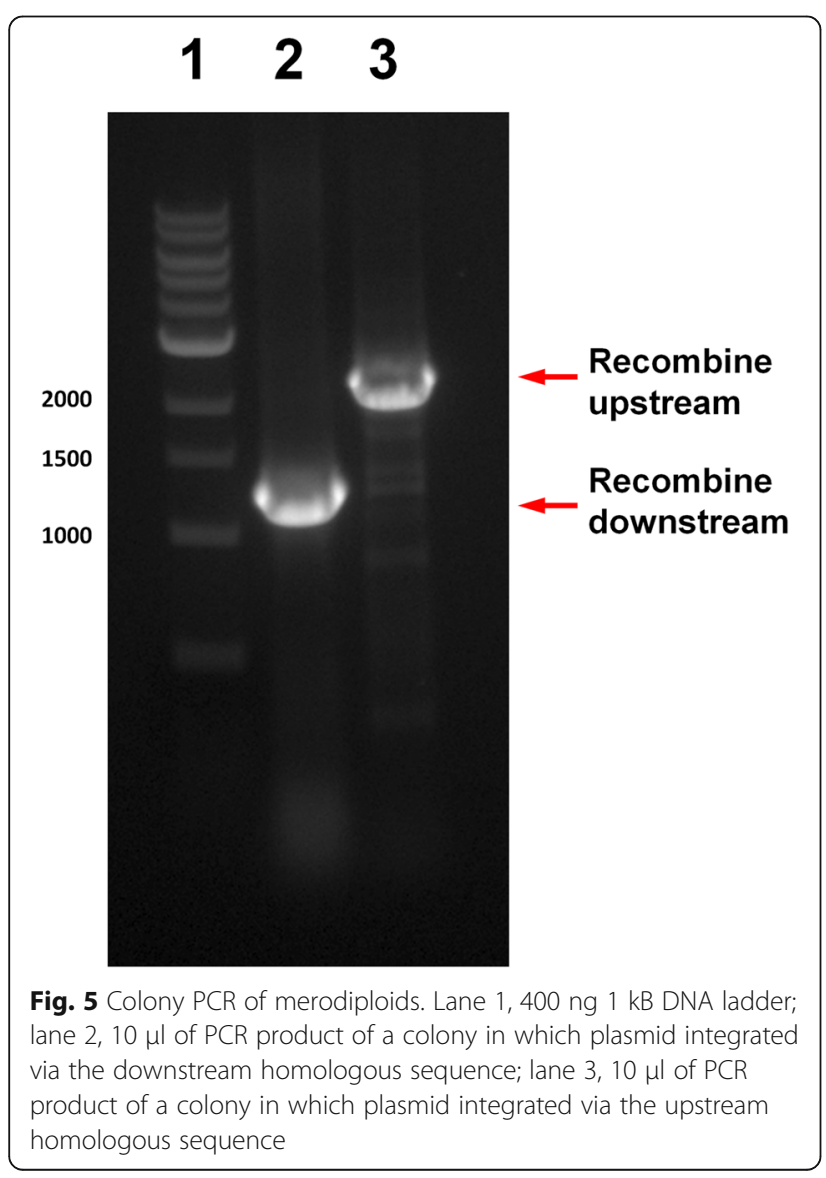

either of these crossover events are illustrated in Fig. 4. If the PCR was performed with the universal pEX18 vector reverse primer (primer 2) and a primer specific to a genomic sequence before the 500 bp upstream (primer 3), reversed patterns would be expected. Generally, 5-10 tetracycline resistant colonies were obtained, all of which were confirmed to be merodiploids by PCR.

\section{Improved sucrose-sacB counter-selection of knockout mutants}

Following confirmation by colony PCR, merodiploids were isolated by streaking selected colonies onto a fresh tetracycline selection plate to avoid carryover contamination. Isolated colonies were then streaked onto TYS10 plates containing $300 \mathrm{mM}$ sucrose to select for colonies that have lost the integrated plasmid backbone containing $s a c B$. The $s a c B$ gene was originally isolated from $B a$ cillus subtilis and encodes levansucrase, which catalyzes the conversion of sucrose to levans, a molecule that is toxic to $P$. aeruginosa. The sucrose intolerance of merodiploids expressing $s a c B$ is caused by accumulation of levans in the periplasmic space, which is a slow process and does not cause immediate lethality. Direct streaking of tetracycline resistant colonies onto TYS10 plates and the alternative streaking from an intermediate culture in LB were directly compared and no difference was observed. However, considering the possibility that a wild type revertant may gain a growth advantage in an intermediate culture and outcompete the deletion mutant, 


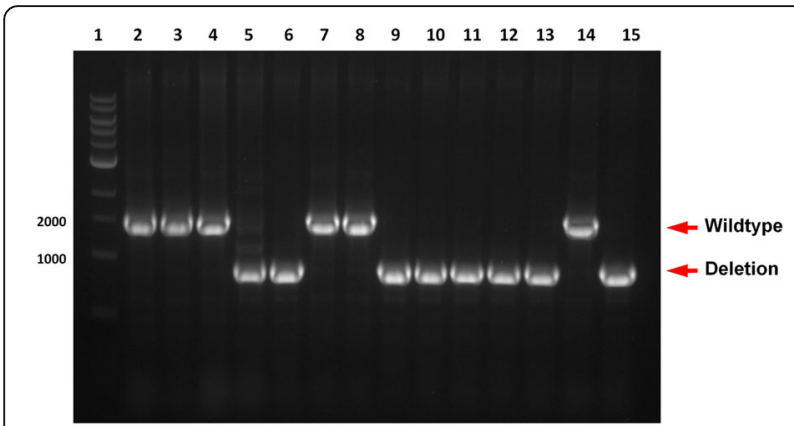

Fig. 6 Screen of deletion mutants by colony PCR. Lane 1, $400 \mathrm{ng} 1 \mathrm{~kb}$ DNA ladder; lane 2-15, $10 \mu \mathrm{l}$ of PCR products of 14 colonies randomly selected from the TYS10 plate. Of the fourteen randomly selected

colonies, 8 colonies were deletion mutants while six colonies reverted to wild type during the second recombination event

streaking an isolated tetracycline resistant colony directly onto a TYS10 plate minimizes undesirable population bias arising prior to counter-selection. The TYS10 medium contains no salt and thus increases the sensitivity of cells to the accumulation of levans [16]. The $s a c B$ counter-selection was performed at room temperature to reduce sucrose hydrolysis and slow down the growth of $P$. aeruginosa to allow more effective selection of merodiploids having undergone the second homologous recombination event.

Sucrose-resistant colonies were analyzed by colony PCR. As illustrated in Fig. 4, two populations formed: one included the desired deletion mutants while the other included wild type revertants. The theoretical ratio between two populations is 1:1 and in the case of hasS, the ratio of $\Delta$ has $S$ vs wild type of 14 randomly selected colonies was 8:6, as judged by the $2 \mathrm{~kb}$ versus $1 \mathrm{~kb}$ insert, respectively (Fig. 6). However, the ratio may be biased towards one population if for example the gene to be deleted is beneficial for bacterial growth. The deletion of has was confirmed by DNA sequencing (Additional file 3).

\section{Discussion}

In this study, we present a rapid and efficient method to create unmarked genetic knockouts of $P$. aeruginosa. In comparison to the previous two-step allelic exchange procedure, which usually takes a minimum of two weeks, this protocol can be completed in one week. Even when compared to the Gateway recombination method described by Choi et al. [9], the time required in the current protocol is reduced and does not depend on proprietary reagents and reengineering of the suicide vector to make it Gateway compatible.

The traditional procedure requires several extra subcloning steps to create the deletion construct, which is complicated by dependence on available restriction sites and the potential for low DNA recovery from agarose gels. In our method, the suicide vector can be simply linearized by any restriction site within the multiple cloning site. The amplified genomic DNA fragments can then be assembled with the linearized vector in one step by Gibson assembly, which is straightforward to set up and does not require any expensive proprietary reagents or instruments. The accuracy of Gibson assembly combined with the desirable construct being obtained with $95 \%$ efficiency $(n=28)$ makes this a viable and attractive alternative to previous methods.

In the engineered gene deletion constructs, the amplified genomic DNA sequence is fused exactly as designed without introducing any other changes to the sequence. The seamless fusion allows for in-frame deletion and leaves no residual nucleotides behind, minimizing polar effects, which is especially important when studying polycistronic operons. The precision of the method is exemplified in the vreA and vreI constructs where the stop codon of vreA and the start codon of vreI overlap. The current method allows for the generation of the $v r e A$ and vreI deletion mutants to single base pair precision while maintaining the start and stop codon of the respective genes (see Additional file 2).

The transformation of the mutant allele construct into $P$. aeruginosa by electroporation provides a fast and straightforward alternative to conjugation. Repetitive microbiological procedures are minimized, reducing workload while greatly reducing the time to complete the two-step allelic exchange. Moreover, direct deletion allele transfer without having to passage $P$. aeruginosa repetitively, as required following conjugation, may reduce the opportunity for introduction of suppressor mutations.

Besides construction of $P$. aeruginosa deletion mutants, this method can also be used to create insertion mutants and locus replacement mutants. Moreover, the protocol may be readily adapted for other related organisms. The fast and streamlined procedure also allows for construction of a panel of mutants in a reasonable timeframe, which can greatly increase the throughput of reverse genetics research.

\section{Conclusion}

Pseudomonas aeruginosa represents a model organism for biological study of biofilm formation and quorum sensing. However, genetic manipulation in $P$. aeruginosa traditionally requires multiple cloning steps in addition to several selective passages following conjugation. Herein we describe a rapid and efficient means of generating deletion mutants using Gibson assembly and direct transformation removing several steps of DNA manipulation and passaging, reducing the likelihood of introducing mutations and without the need for proprietary reagents. 


\section{Additional files}

Additional file 1: Oligonucleotide primers used in this study. (DOCX $71 \mathrm{~kb}$ )

Additional file 2: Sequencing results for the vreA (A) and vrel (B) deletion strains. (DOCX 10162 kb)

Additional file 3: Sequencing results for the hasS deletion mutant. (DOCX $5552 \mathrm{~kb})$

\section{Abbreviations}

BHI: Bovine heart infused media; ECF: Extra cytoplasmic function; has: Heme assimilation system; LB: Luria broth; PCR: Polymerase chain reaction; TB: Terrific broth; TYS10: Tryptone, yeast extract, sucrose broteh; vre: Virulence regulator involved ECF system

\section{Acknowledgements}

We thank Dr. Susana Mouriño for helpful discussions, Angela Nguyen, Bennett Giardina and Alexandria Reinhart for sharing plasmids.

\section{Funding}

This work was funded by the National Institute of Health (NIH) Al102883 to AW.

\section{Availability of data and materials}

Not applicable

\section{Authors' contributions}

AW and WH conceived experiments. WH designed and performed al experiments. WH wrote the manuscript and AW edited the final version. Both authors read and approved the final manuscript.

\section{Ethics approval and consent to participate}

Not applicable

\section{Consent for publication}

Not applicable

\section{Competing interests}

The authors declare that they have no competing interests.

Received: 29 June 2017 Accepted: 13 September 2017

Published online: 19 September 2017

\section{References}

1. Antibiotic Resistance Threats in the United States, 2013. In. Edited by Prevention CDC: US Department of Health and Human Services, 2013: 1-114

2. Giske CG, Monnet DL, Cars O, Carmeli Y. Clinical and economic impact of common multidrug-resistant gram-negative bacilli. Antimicrob Agents Chemother. 2008:52(3):813-21.

3. Stover CK, Pham XQ, Erwin AL, Mizoguchi SD, Warrener P, Hickey MJ, Brinkman FS, Hufnagle WO, Kowalik DJ, Lagrou M, et al. Complete genome sequence of Pseudomonas aeruginosa PAO1, an opportunistic pathogen Nature. 2000:406(6799):959-64.

4. Quenee L, Lamotte D, Polack B. Combined sacB-based negative selection and cre-lox antibiotic marker recycling for efficient gene deletion in Pseudomonas aeruginosa. BioTechniques. 2005;38(1):63-7.

5. Schweizer HP. Allelic exchange in Pseudomonas aeruginosa using novel ColE1-type vectors and a family of cassettes containing a portable oriT and the counter-selectable Bacillus subtilis sacB marker. Mol Microbiol. 1992:6(9):1195-204.

6. Hmelo LR, Borlee BR, Almblad H, Love ME, Randall TE, Tseng BS, Lin C, Irie Y, Storek KM, Yang JJ, et al. Precision-engineering the Pseudomonas aeruginosa genome with two-step allelic exchange. Nat Protoc. 2015;10(11):1820-41.

7. Hoang TT, Karkhoff-Schweizer RR, Kutchma AJ, Schweizer HP. A broad-hostrange Flp-FRT recombination system for site-specific excision of chromosomally-located DNA sequences: application for isolation of unmarked Pseudomonas aeruginosa mutants. Gene. 1998;212(1):77-86.

8. Choi KH, Schweizer HP. An improved method for rapid generation of unmarked Pseudomonas aeruginosa deletion mutants. BMC Microbiol. 2005:5:30
9. Gibson DG, Young L, Chuang RY, Venter JC, Hutchison CA 3rd, Smith HO. Enzymatic assembly of DNA molecules up to several hundred kilobases. Nat Methods. 2009;6(5):343-5.

10. Holloway BW. Genetic recombination in Pseudomonas Aeruginosa. J Gen Microbiol. 1955;13(3):572-81.

11. Choi KH, Kumar A, Schweizer HP. A 10-min method for preparation of highly electrocompetent Pseudomonas aeruginosa cells: application for DNA fragment transfer between chromosomes and plasmid transformation. Jicrobiol Methods. 2006;64(3):391-7.

12. Diver JM, Bryan LE, Sokol PA. Transformation of Pseudomonas aeruginosa by electroporation. Anal Biochem. 1990;189(1):75-9.

13. Farinha MA, Kropinski AM. High efficiency electroporation of Pseudomonas aeruginosa using frozen cell suspensions. FEMS Microbiol Lett. 1990;58(2):221-5.

14. Smith AW, Iglewski BH. Transformation of Pseudomonas aeruginosa by electroporation. Nucleic Acids Res. 1989;17(24):10509.

15. Wolfgang MC, Lee VT, Gilmore ME, Lory S. Coordinate regulation of bacteria virulence genes by a novel adenylate cyclase-dependent signaling pathway. Dev Cell. 2003;4(2):253-63.

16. Blomfield IC, Vaughn V, Rest RF, Eisenstein BI. Allelic exchange in Escherichid coli using the Bacillus subtilis sacB gene and a temperature-sensitive pSC101 replicon. Mol Microbiol. 1991:5(6):1447-57.

17. Chang DC. Guide to electroporation and electrofusion. CA, USA: Academic Press; 1992.

\section{Submit your next manuscript to BioMed Central and we will help you at every step:}

- We accept pre-submission inquiries

- Our selector tool helps you to find the most relevant journal

- We provide round the clock customer support

- Convenient online submission

- Thorough peer review

- Inclusion in PubMed and all major indexing services

- Maximum visibility for your research

Submit your manuscript at www.biomedcentral.com/submit
Biomed Central 